\title{
Influence of Control Time Delay on the Dynamics of Satellite Capture
}

\author{
By Hiroki NAKANISHI, Masaaki KODAMA and Kazuya YOSHIDA
}

\begin{abstract}
Department of Aerospace Engineering, Tohoku University, Sendai, Japan (Received May 2nd, 2008)

One of the most important phases of satellite servicing operations by a space robot is the "contact" phase. During the contact between the end-effector and grasping point, there is a risk that the target and the robot can be pushed away from each other by the contact force. Therefore, the method to control the end-effector by the impedance control so as not to deflect the target was suggested. However, all of real control systems have the control time delay. In case of the target capture operation, the time delay of the impedance control makes the impulse of the contact larger. In this paper, to verify the effect of the time delay of the impedance control, experiments with a air-floating testbed and numerical simulations are carried out.
\end{abstract}

Key Words: Satellite Capture, Contact Dynamics, Impedance Control, Control Time Delay

\section{Nomenclature}

$\phi \quad$ : joint angle

$\tau \quad$ : joint torque

$\boldsymbol{F}_{e} \quad$ : external force on hand

c : nonlinear velocity term

$\boldsymbol{J}^{*} \quad$ : generalized Jacobian matrix ${ }^{1)}$

$\boldsymbol{H}^{*} \quad$ : generalized inertia matrix ${ }^{2)}$

$\boldsymbol{x} \quad$ : position

$M$ : mass characteristic

$D$ : viscosity characteristic

$\boldsymbol{K}$ : stiffness characteristic

$m_{0}$ : mass

$f_{e} \quad$ : uniaxial force on hand

$k \quad:$ uniaxial stiffness characteristic

$d \quad$ : uniaxial viscosity characteristic

$x \quad$ : uniaxial position

$\delta \quad:$ deformation

$f_{s} \quad$ : friction force between probe shaft and holder

Subscripts

0 : chaser base

h : chaser hand

t : target

i : arm impedance

c : contact surface

s : contact probe on air floating testbed

\section{Introduction}

The importance of orbital infrastructures (ex. satellite, space station, etc.) is increasing. Currently the construction and maintenance of such infrastructures are done by EVA (External Vehicle Activity) of astronauts. However, these operations are dangerous and expensive. Hence, there are great hopes that unmanned space robots will perform such servicing missions instead of astronauts.

One of the most important phases of such satellite servicing operations by a space robot is the "contact" phase. During the contact between the end-effector and grasping point, there is a risk that the target and the robot can be pushed away from each other by the contact force.

An on-orbit demonstration of robotics and rendezvous-docking technologies was carried out by ETS-VII of NASDA, Japan from 1997 to $1999{ }^{3)}$. In this demonstration, a sub-satellite (target) was approached and successfully docked with by a main robotic satellite (chaser). However, the target was equipped with dedicated docking instruments (grapple fixture, optical marker, etc.) fitting for the capture instruments of the chaser satellite. These equipments prevented the target from being pushed away due to the collision forces when the end-effector and the grapple fixture come into contact. Such target is called "cooperative." In contrast, the satellites already existing in orbit and requiring service are "noncooperative" targets with no such features.

In case of capturing a non-cooperative target, the contact force should be properly controlled in order to prevent the space robot from pushing the target away. It is well known that utilization of impedance control for manipulators is one of the most reasonable methods to control the contact force. The target velocity after the contact depends on the impedance of the end tip of the manipulator. If the manipulator impedance is smaller than the mass of the target, the post-impact escape velocity of the target is smaller than the velocity of the manipulator 4). That means the contact is maintained after the initial contact. Hence, control the impedance of the manipulator with accuracy is key issue for the safety target capture. One of the difficulties for such accurate control is the control time delay problem. When there is a time delay in a control sequence of the manipulator, the apparent impedance can be different 


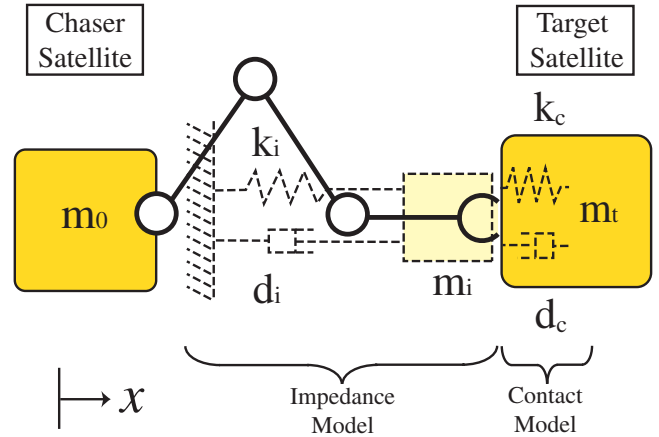

Fig. 1. Satellite capture model

from the commanded value ${ }^{5)}$. The control time delay is unavoidable in all control systems fatefully. In case of spacecrafts especially, it is more apparent because the reliability of the processors have priority over their performance. However, the influence of the delay on the contact dynamics at the target capture has not been clarified precisely yet.

In this paper, the contact dynamics at a satellite capture with a control time delay is discussed. An orbital satellite servicing robot with a manipulator arm under impedance control is assumed. The dynamics model of the manipulator including control time delay is formulated. The validity of the model is tested by a micro-gravity experiment using an air-floating testbeds and a manipulator arm. Using these models, the influence of the impedance of the arm and the control time delay on the contact force is evaluated.

\section{Contact Dynamics of Space Robot}

\subsection{Satellite capture model}

Figure 1 shows a uniaxial component model in which two satellites come into contact. The robot satellite on the left has a manipulator arm that allows impedance control attached to a base of mass $m_{0}$. The satellite on the right is the target of capture that is modeled as a floating rigid body of mass $m_{t}$. When the robot hand is in contact with the target, mechanical impedance (contact impedance) is defined between both parties.

The present paper considers the influences of the impedance and the control time delay of the robot hand on the motion of the target using this model.

\subsection{Impedance control for free-flying space robot}

The equation of motion of a free-floating space robot is expressed by Eq. (1).

$$
\boldsymbol{\tau}=\boldsymbol{H}^{*} \ddot{\boldsymbol{\phi}}-\boldsymbol{J}^{* T} \boldsymbol{F}_{e}+\boldsymbol{c}
$$

Impedance control of the robot arm allows the inertial characteristics of the hand to vary over a wide range.

The impedance characteristics $\boldsymbol{M}_{i}, \boldsymbol{D}_{i}$, and $\boldsymbol{K}_{i}$ are assigned as shown in Eq. (2). Here, the orientation of the hand is excluded for simplicity.

$$
\boldsymbol{M}_{i} \ddot{\boldsymbol{x}_{h}}+\boldsymbol{D}_{i} \Delta \dot{\boldsymbol{x}_{h}}+\boldsymbol{K}_{i} \Delta \boldsymbol{x}_{h}=\boldsymbol{F}_{e}
$$

where, $\Delta \boldsymbol{x}_{h}=\boldsymbol{x}_{h}-\boldsymbol{x}_{h d}$ is the deformation from the equilibrium point of the impedance control $\boldsymbol{x}_{h d}$. The joint torque used to realize such impedance characteristics is theoretically obtained by generalized Jacobian and Eq. (3) ${ }^{6)}$.

$$
\begin{aligned}
\boldsymbol{\tau}= & \boldsymbol{H}^{*} \boldsymbol{J}^{*-1}\left\{\boldsymbol{M}_{i}^{-1}\left(\boldsymbol{F}_{e}-\boldsymbol{D}_{i} \Delta \dot{\boldsymbol{x}}_{h}-\boldsymbol{K}_{i} \Delta \boldsymbol{x}_{h}\right)\right\} \\
& \left.-\dot{\boldsymbol{J}}^{*} \dot{\boldsymbol{\phi}}-\ddot{\boldsymbol{x}}_{g h}\right\}-\boldsymbol{J}^{* T} \boldsymbol{F}_{e}+\boldsymbol{c}
\end{aligned}
$$

\section{Relation Between Contact Motion and Impedance}

The collision motion after the initial contact is classified into following three states with the initial hand velocity $\dot{x}_{h}$ and post impact target velocity $\dot{x}_{t}^{\prime}$

1. The target is pushed away. $\left(\dot{x}_{t}^{\prime}>\dot{x}_{h}\right)$

2. The relative velocity between the hand and the target become $0 .\left(\operatorname{dot} x_{t}^{\prime}=\dot{x}_{h}\right)$

3. The hand is pushed back by the target. $\left(\dot{x}_{t}^{\prime}<\right.$ $\left.\dot{x}_{h}\right)$

These phenomena depend on the impedance of the hand. In general, The motion after the initial contact is changed from 3 to 1 in proportion as the magnitude of the impedance. The state 1 should be avoided in order to make the capture a success.

Using an equivalent mass model, the relation between the target motion and the hand impedance can be explained.

The equivalent mass $m_{v}$ of the hand with an impedance is defined as ${ }^{7)}$ :

$$
m_{v}=\frac{F_{e}}{\ddot{x}_{h}}
$$

In practice, the equivalent mass varies while the contact. In this paper, let the equivalent mass is redefined as:

$$
m_{v}=\frac{1}{T} \int_{0}^{T} \frac{F_{e}}{\ddot{x}_{h}} d t
$$

where $T$ is the contact time of one collision. The relationship between $m_{v}$ and the impedance characteristics can be expressed as following:

$$
m_{v}=m_{i}+\alpha
$$

where $\alpha$ is a term depending on the stiffness $k_{i}$ and viscosity $d_{i}$. Using the above equivalent mass model, 


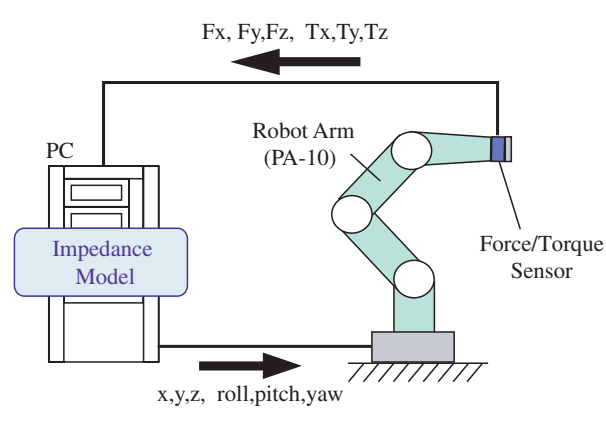

(a) Control sequence of robot arm

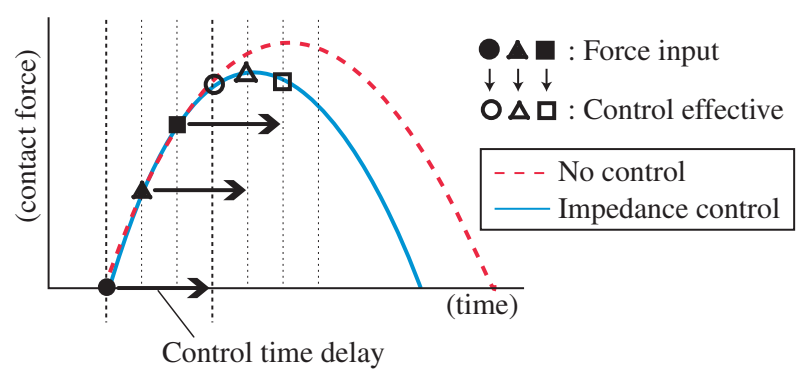

(b) Influence of control time delay on force feedback

Fig. 2. Schematic of control time delay

the chaser's hand can be modeled as a virtual point mass $m_{v}$. Consider the case in which the hand with initial velocity $\dot{x}_{h}$ collides with the target with velocity $\dot{x}_{t}$. Then the post-collision velocity $\dot{x}_{t}^{\prime}$ can be calculated using the coefficient of restitution $e$ as follows ${ }^{4)}$ :

$$
\dot{x}_{t}^{\prime}=\frac{m_{v} \dot{x}_{h}+m_{t} \dot{x}_{t}+m_{v} e\left(\dot{x}_{h}-\dot{x}_{t}\right)}{m_{v}+m_{t}}
$$

\section{Control Time Delay}

The sequence of impedance control of the manipulator arm is shown in Fig. 2-(a). The control time delay occurs because of digitalization time of data from a force-torque sensor, data communication time and calculation time for objective position. This depends on performance of its systems, but any control system has time delay necessarily.

Figure 2-(b) shows a relation of contact force and time delay of impedance control. Because of the control time delay, it takes a little time from the chaser receiving force inputs to starting the motion according to the impedance control. The chaser under no control continues to push the target during the period. In general, that means the apparent impedance of the hand comes to the specific one of the chaser arm at the servo lock state.

In following sections, the relation between the contact force, impedance characteristics, and control

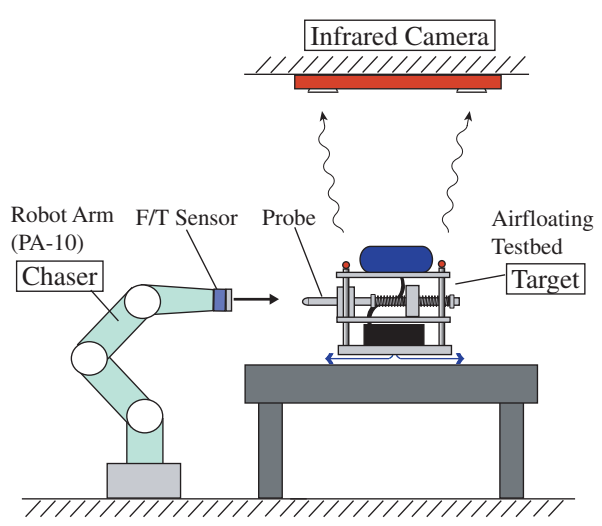

Fig. 3. Experimental setup

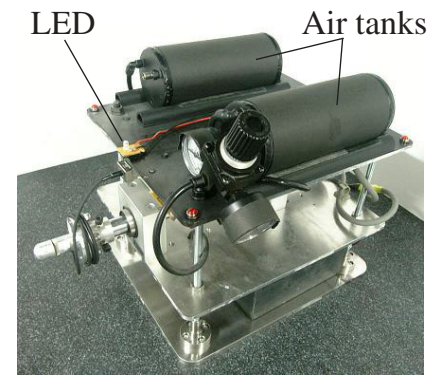

(a) All view

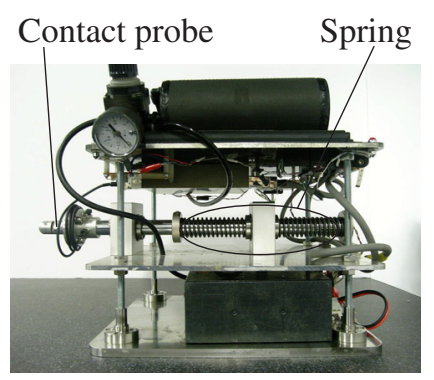

(b) Side view
Fig. 4. Airfloating Testbed (Target)

time delay is investigated through the experiments and simulations.

\section{Experimental Setup}

Figure 3 shows the schematic of the experimental setup. A 7-DOF manipulator arm (Mitsubishi Heavy Industries, PA-10) is used as a chaser's manipulator. A force-torque sensor mounted on the end tip of the chaser measures the contact forces and moments, and impedance control is applied. The control system includes a fatal time delay of 30 [ms], which is measured in the preliminary experiments.

The air-floating testbed as a target is shown in Fig. 4. A miniature model of a spacecraft was set on a precision furface plate. The testbed floats on the plate supported by a thin layer of air leaking from the bottom of the model. The friction between the precision surface plate and the bottom of the spacecraft model becomes nearly zero. Hence, a micro-gravity environment in two dimensions is obtained. Flotation for 1 min can be acieved using two air tanks (maximum pressure of $0.9[\mathrm{MPa}])$ mounted on the testbed. A shaft is mounted on the center of the testbed as a contact probe to the end tip of the chaser. The contact probe has an exchangeable spring at its base for changing the mechanical impedance of the contact point. In order to obtain the positional data of the 


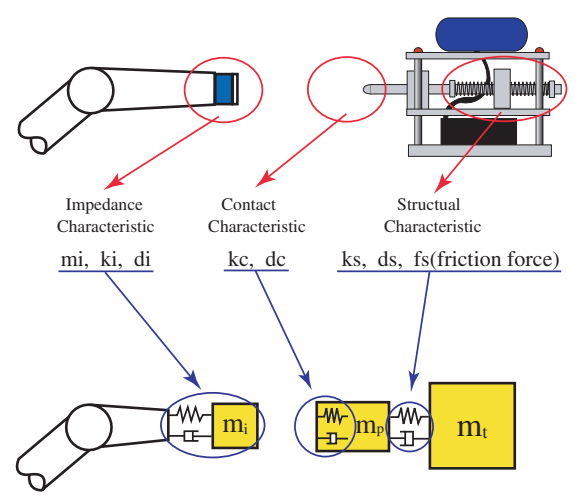

Fig. 5. Contact model of the experimental setup

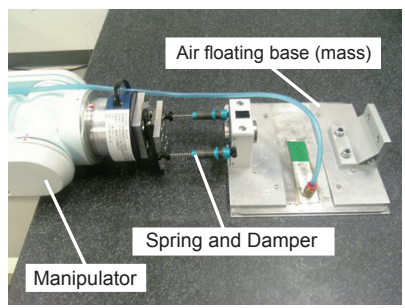

Fig. 6. Mechanical impedance structure

testbed, an infrared camera is placed on the ceiling over the experimental setup to track infrared LEDs on the testbed.

\subsection{Simulation model with control time de- lay}

In order to investigate the effects of control time delay, a simulation model based on a linear springdamper model is constructed. Figure 5 is the dynamics model in the simulation. The contact force $F_{e}$ is given by following equation:

$$
F_{e}=-k_{c} \delta_{c}-d_{c} \dot{\delta}_{c}
$$

The external force applied to the target main body $f$ is given by the following equation:

$$
f=-k_{s} \delta_{s}-d_{s} \dot{\delta}_{s}+\operatorname{sgn}\left(\delta_{s}, f_{s}\right)
$$

To replicate the control time delay, an impedance controlled input is not used in the next step of the dynamics simulation but instead in the time delay step at the end of the dynamics simulation. Further, the feedback control gain is determined by comparing the experimental results and simulation results.

\subsubsection{Verification of simulation model}

Here, In order to verify the validity of the simulation model and to estimate the contact parameters, the result of the simulation and experiment are compared. Furthermore, to compare with the results of the no time delay case, a mechanical impedance structure is developed (Fig. 6.). It is attached to the end tip of the arm, and supplies the arm impedance.

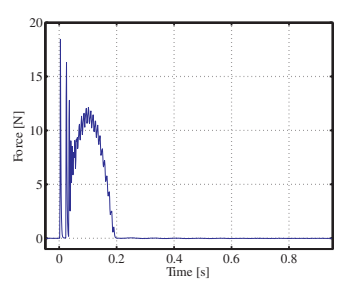

(a) Experiment

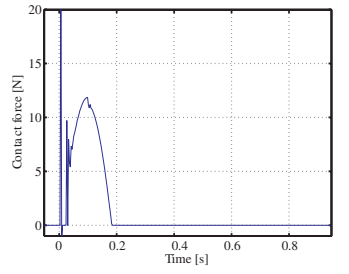

(b) Simulation
Fig. 7. Contact force (Impedance control)

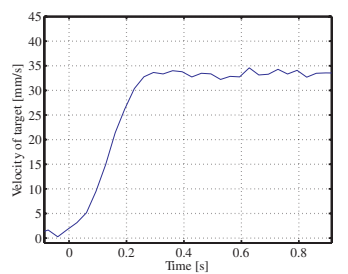

(a) Experiment

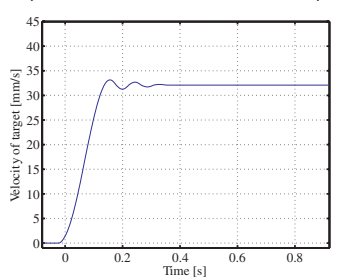

(b) Simulation
Fig. 8. Target velocity (Impedance control)

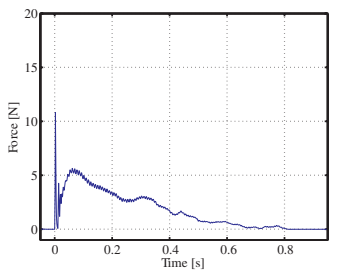

(a) Experiment

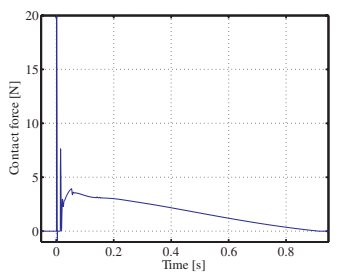

(b) Simulation
Fig. 9. Contact force (Impedance mechanism)

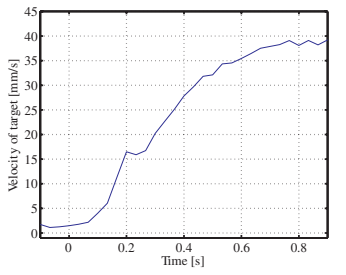

(a) Experiment

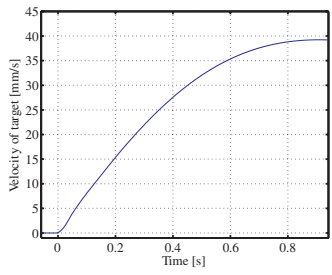

(b) Simulation
Fig. 10. Target velocity (Impedance mechanism)

The conditions of the chaser were as follows: the impedance characteristics of the arm were $m_{i}=1.8$ $[\mathrm{kg}], k_{i}=315[\mathrm{~N} / \mathrm{m}]$, and $d_{i}=120[\mathrm{~N} /(\mathrm{m} / \mathrm{s})]$ in both cases. Further, $m_{t}=44.2[\mathrm{~kg}], m_{p}=1.25[\mathrm{~kg}], k_{s}$ $=6300[\mathrm{~N} / \mathrm{m}], d_{s}=30[\mathrm{~N} /(\mathrm{m} / \mathrm{s})]$, and $f_{s}=0.35$ [N]. The time delay of the impedance control was 30 [ms], and the contact parameters were determined to be $k_{c}=750000[\mathrm{~N} / \mathrm{m}]$ and $d_{c}=300[\mathrm{~N} /(\mathrm{m} / \mathrm{s})]$ by employing a frequency analyses of the contact force.

Figures $7-10$ show the results of the experiment and simulation under the same conditions. In the case of the impedance control (with time delay), the peak of the contact force is higher and the contact time is shorter than those in the case of the impedance mechanism (without time delay). The simulation results correspond to these experimental results well. Therefore, it can be concluded that the 
simulation model including control time delay sufficiently represents a real collision.

\section{Influence of Impedance on Contact with- out Time Delay}

\subsection{Relation between impedance and con- tact motion}

In order to clarify the influence of time delay on the hand impedance and the contact force, The relationship between pure impedance and the contact motion of the target in the case of no time delay is investigated using simulations.

The chaser arm with impedance approaches the target with a constant velocity. The contact force and target velocity vary with the impedance. If the target velocity after the initial contact is smaller than the approaching velocity of the arm, the contact can be maintained. The contact force and the target velocity at the initial contact are shown in Fig. 11. The approach velocity is $30[\mathrm{~mm} / \mathrm{s}]$. The impedance ratio of $m_{i}, d_{i}$, and $k_{i}$ are constant at 1:100:2, and the proportional constant determines the impedance characteristics.

The higher impedance, increases the contact time and target velocity after the collision. In the proper impedance case, the target velocity becomes identical to the approach velocity of the chaser arm. This implies that the chaser arm maintains the contact after the initial one. In the higher impedance case, the target is pushed away from the arm. In the lower impedance case, the contact is repeated, and the target velocity eventually reaches the approach velocity.

\subsection{Influence of each impedance characteris- tics on contact}

One of the evaluation index of the impedance for the contact motion is given by Eq. (6). $\alpha$ is a term depending on the $k_{i}$ and $d_{i}$. This implies that the dominant factor of the impedance for the contact dynamics is mass characteristic. In this section, the influence of $k_{i}$ and $d_{i}$ is investigated. Figure 12 shows the relation between the target velocity after the initial contact and these characteristics. From this result, the viscosity characteristic $d_{i}$ is the dominant factor for the contact motion. On the other hand, the influence of the stiffness $k_{i}$ is minor. Note that it may be appropriate to think that $d_{i}$ is makes the contact stable. However, this result shows the impact increases with viscosity.

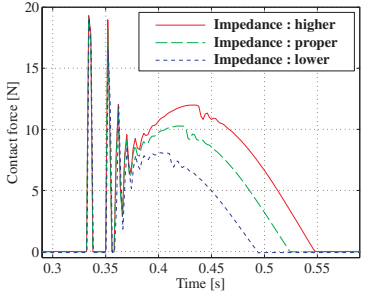

(a) Contact force at initial one

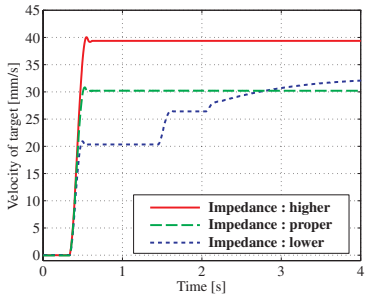

(b) Target velocity
Fig. 11. Relation between impedance and contact motion

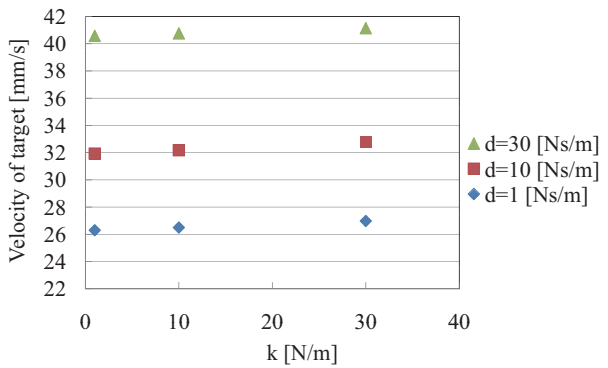

Fig. 12. Relation between $k, d$, and target velocity after contact

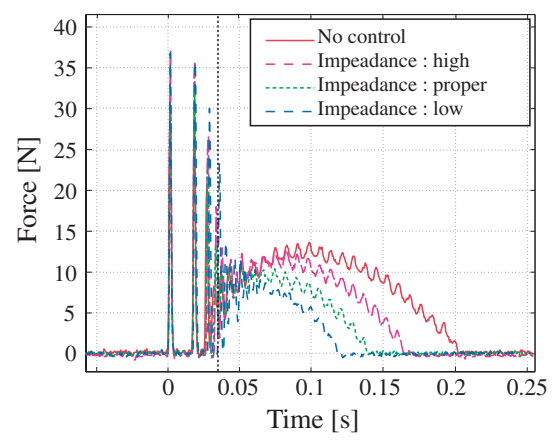

Fig. 13. Contact force variation with hand impedance (with time delay: $30[\mathrm{~ms}]$ )

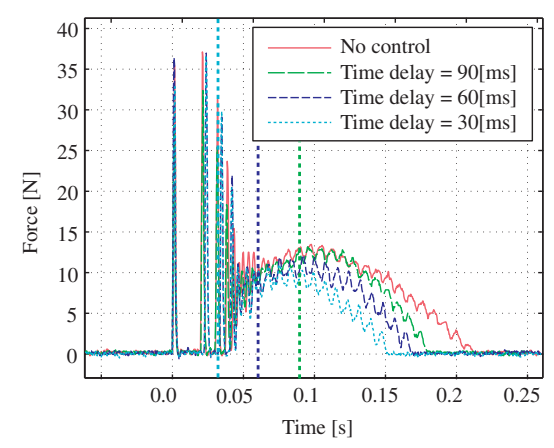

Fig. 14. Contact force variation with time delay

\section{Influence of Impedance on Contact with Time Delay}

\subsection{Influence of time delay on contact}

Contact experiments are conducted to investigates the effects of control time delay on the contact force 


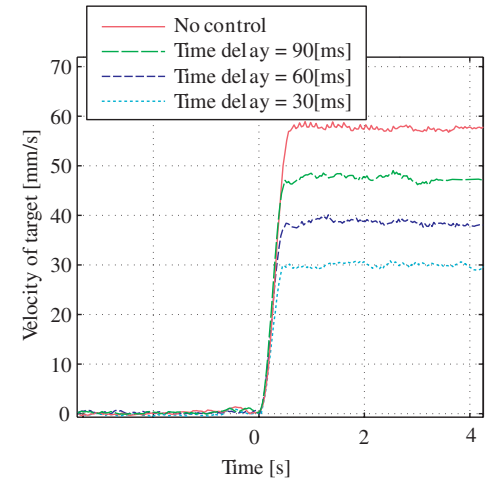

Fig. 15. Target velocity variation with time delay

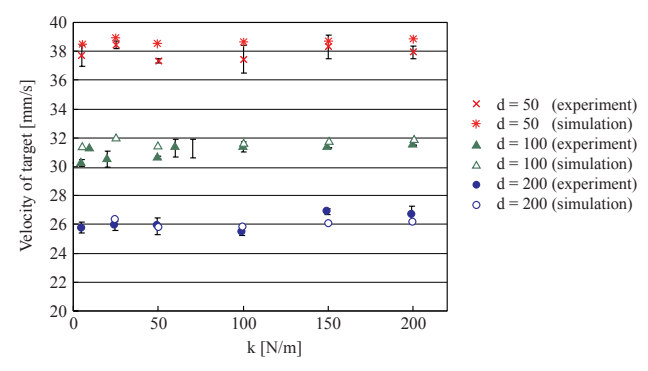

Fig. 16. Relation between $k, d$, and target velocity after contact (with time delay: 30 [ms])

and motion of the chaser. The experimental conditions are identical to the above experiments and simulations. The variation of the initial contact forces for a time delay of $30[\mathrm{~ms}]$ are shown in Fig. 13 . The waveform of each contact force changes from approximately $30[\mathrm{~ms}]$ on the basis of each of the impedances described in the section 6 . The control time delay of this system is $30[\mathrm{~ms}]$. The reasons for these results are as follows: In the case of any of the impedance characteristics given by the control, the specific impedance of the arm structure appears during the control time delay because the control effect has not occurred. Hence, the total impulse of the chaser during the control time delay is constant for all impedances. In such case, the $m_{v}$ in Eq. (6) is the time average of the one under time delay and the one under impedance control.

Figure 14 shows the initial contact forces when the control time delay is approximately 30,60, and 90 [ms]. The controlled impedance characteristics is constant. The line "No control" is in the case that the arm is servo locked. In each case, the waveform of the contact force is same as the "No control" case until end time of the delay and then vary as to each characteristics respectively.

Figure 15 shows the profiles of the target velocity. As the time delay increases, the contact time and target velocity after collision increase. Therefore, as the time delay increases, the total impulse to the target during the time delay of each case increases. By comparing the wave forms of Figs. 13. and 15., the effect of delay time increase is similar to the effect of impedance increase. In this case, an increase in delay time by $60[\mathrm{~ms}]$ is equivalent to ten times the magnification ratio of the impedance.

\subsection{Influence of each impedance characteris- tics on contact}

The influence of $k_{i}$ and $d_{i}$ when the control time delay exists is also investigated. Figure 16 shows the relationship between the target velocity after the initial contact and these characteristics. The viscosity characteristic $d_{i}$ is the dominant factor for the contact motion as well as the no-delay case.

\section{Conclusion}

In this paper, the contact dynamics for capturing free-flying target by robot arm under impedance control with control time delay was analyzed. The experimental setup using an air-floating testbed and a simulation model was conducted to investigate the relationship between the impedance and the contact force and motion. The dominant factor of impedance for contact force was clarified. The influence of the control time delay on the contact force was also discussed.

\section{References}

1) Umetani, Y. and Yoshida, K.: Resolved Motion Rate Control of Space Manipulators with Generalized Jacobian Matrix, IEEE Trans. on Robotics and Automation, 5 (1989), pp. 303-314.

2) Yoshida, K., Kurazume, R., Sashida, N. and Umetani, Y.: Modeling of Collision Dynamics for Space FreeFloating Links with Extended Generalized Inertia Tensor, Proc. 1992 IEEE Int. Conf. on Robotics and $\mathrm{Au}-$ tomation, 1992, pp. 899-904.

3) Kasai, T., Oda, M. and Suzuki, T.: Results of the ETS-7 Mission - Rendezvous Docking and Space Robotics Experiments, Proc. 5th Int. Symp. on AI, Robotics and Automation in Space, iSAIRAS'99, 1999, pp. 299-306.

4) Yoshida, K., Nakanishi, H., Ueno, H., Nishimaki, T. and Oda, M.: Dynamics, control and impedance matching for robotic capture of a non-cooperative satellite, Advanced Robotics, 18 (2004), pp. 175-198.

5) Nakanishi, H., Yoshida, K. and Tanaka, D.: Contact Dynamics Analysis of a Space Craft in Micro Gravity with an Air-floating Testbed, Proc of the 25th International Symposium on Space Technology and Science (Selected papers), 2006, pp. 792-795.

6) Nakanishi, H. and Yoshida, K.: Impedance Control of Free-Flying Space Robot for Orbital Servicing, Journal of Robotics and Mechatronics, 18 (2006), pp. 608-617.

7) The Japan Society of Mechanical Engineers : JSME Mechanical Engineers' Hand book, Fundamentals, $\alpha 2$ Dynamics of Machinery, 2004. 